Covidien. 6; C; Medtronic, Covidien, Stryker, Neuravi, BrainsGate, Pfi zer, Bristol-Myers Squibb, Boehringer Ingelheim.

\section{P-011 IMAGING AND HISTOPATHOLOGY OF THROMBI IN ACUTE ISCHEMIC STROKE: SYSTEMATIC REVIEW AND META-ANALYSIS}

${ }^{1}$ W Brinjikji, ${ }^{2} S$ Duffy, 'D Kallmes. 'Radiology, Mayo Clinic, Rochester, MN; ${ }^{2}$ GallwayMayo Institute of Technology, Gallway, Ireland

\subsection{6/neurintsurg-2016-012589.53}

Background and purpose There has been growing interest in the imaging and histopathology of retrieved thrombi after the introduction of mechanical thrombectomy for the treatment of acute ischemic stroke. We conducted a systematic review and meta-analysis of imaging and histological characteristics of thrombi in acute ischemic stroke.

Materials and methods We identified all studies published between January 2005 and December 2015 that reported findings related to the histological and/or imaging characteristics of thrombi in patients with acute ischemic stroke secondary to large vessel occlusion. The five outcomes examined in this study were 1) the association between histological composition of thrombi and stroke etiology, 2) the association between thrombi histological composition and angiographic outcomes, 3 ) the association between imaging and histological characteristics of thrombi in stroke, 4) the association between imaging characteristics of thrombi and angiographic outcomes and 5) the association between imaging characteristics of thrombi and stroke etiology.

Results There was no significant difference in the proportion of RBC rich thrombi between cardioembolic and large artery atherosclerosis etiologies $(\mathrm{OR}=1.62,95 \% \mathrm{CI}=0.1-28.0$, $\mathrm{P}=0.63$ ). Patients with hyperdense artery sign had a higher odds of having RBC rich thrombi than those without a hyperdense artery sign on $\mathrm{CT}(\mathrm{OR}=9.0,95 \% \mathrm{CI}=2.6-31.2$, $\mathrm{P}<0.01)$. Patients with a good angiographic outcome had a mean HU of 53.0 compared to a mean $\mathrm{HU}$ of 47.3 for patients with a poor angiographic outcome $(\mathrm{MSD}=5.6$, $95 \% \mathrm{CI}=1.1-10.0, \mathrm{P}=0.02)$. There was no association between imaging characteristics and stroke etiology $(\mathrm{OR}=$ $1.13,95 \%$ CI $=0.32-4.00, \mathrm{P}=0.85)$.

Conclusions Hyperdense artery sign is associated with RBC rich thrombi and improved recanalization rates. Further research is needed to determine the association between thrombi composition and stroke etiology as well as revascularization outcomes.

Disclosures W. Brinjikji: None. S. Duffy: 5; C; Neuravi LTD. D. Kallmes: None.

\section{P-012 LONGER WORKING LENGTH OF THE SOLITAIRE RETRIEVAL DEVICE IMPROVES REVASCULARIZATION}

W Holloway, I Akhtar, J Halpin, C Martin, N Akhtar. Marion Bloch Neuroscience Institute, Saint Luke's Hospital of Kansas City, Kansas City, MO

\subsection{6/neurintsurg-2016-012589.54}

Introduction/purpose Endovascular treatment using stent retrievers are now the standard of care in patients with acute ischemic stroke due to proximal middle cerebral artery occlusion. Recent randomized clinical trials have shown stent retriever recanalization rates (TICI 2 b or higher) surpassing $80 \%$.
The Solitaire stent retriever is available in different diameters and lengths. More recently, in August 2014, a $4 \times 40$ device was made available for thrombus retrieval in the US. While there have been many studies comparing the Solitaire to the other mechanical thrombectomy devices, at present, there have been little to no human data comparing the relatively new $4 \times 40$ Solitaire device with the older $4 \times 20$ and $4 \times 15$ devices. In our presentation we will be looking at the cases in which the $4 \times 40$ device was deployed and compare the recanalization rates to those achieved with cases treated with the $4 \times 20$ and $4 \times 15$ in patients with acute stroke.

Materials and methods Materials used were the Solitaire Revascularization device in the sizes $4 \times 40 \mathrm{~mm}, 4 \times 20 \mathrm{~mm}$, and $4 \times 15 \mathrm{~mm}$. All devices are identical in diameter but differ in the working length.

Methods This is a retrospective study of 247 stroke patients who underwent clot retrieval using a $4 \mathrm{~mm}$ diameter Solitaire device as the first device deployed in a case at Saint Luke's Hospital of Kansas City from 2012 till January 2016. Of the 247 total patients, 34 had undergone initial endovascular treatment with the Solitaire $4 \times 40$ device. The remainder were either treated with the $4 \times 20$ or the $4 \times 15$ Solitaire devices. Successful recanalization was determined as having a final TICI score of $2 \mathrm{~b}$ or higher ( $>50 \%$ recanalization). Almost every case was performed with a balloon guide catheter inflated in the neck vasculature with aspiration on the guide catheter during Solitaire retrieval.

Results The following Table 1 shows the recanalization rate with the different Solitaire devices.

\begin{tabular}{lll} 
Abstract P-012 Table 1 & & \\
\hline Size of solitaire Device & Number of patients & $\begin{array}{l}\text { Recanalization rate } \\
\text { (TICl 2b or higher) }\end{array}$ \\
\hline $4 \times 15$ & $(\mathbf{n})$ & $84.38 \%$ \\
$4 \times 20$ & 32 & $88.39 \%$ \\
$4 \times 40$ & 181 & $97.06 \%$ \\
\hline P-Value: 0.1087 & 34 &
\end{tabular}

Conclusions The Solitaire $4 \times 40$ device showed a statistical trend for achieving better recanalization compared to the shorter devices. However due to our small sample size of patients undergoing treatment with the $4 \times 40$, further investigation is warranted to determine whether this result is maintained in larger sample size such as the STRATUS registry. Disclosures W. Holloway: None. I. Akhtar: None. J. Halpin: None. C. Martin: None. N. Akhtar: None.

\section{P-013 USE OF THE SOLITAIRE DEVICE FOR EMERGENCY REVASCULARIZATION OF THE SUPERIOR MESENTERIC ARTERY}

${ }^{1} \mathrm{~J}$ Dalfino, ${ }^{1} \mathrm{~A}$ Paul, ${ }^{2} \mathrm{~J} \mathrm{Hnath} .{ }^{1}$ Neurosurgery, Albany Medical Center, Albany, NY; ${ }^{2}$ Albany Medical Center, Albany, NY

\subsection{6/neurintsurg-2016-012589.55}

Background The Solitaire device (Medtronic) was designed for thrombectomy in acute stroke, but its 4-6 $\mathrm{mm}$ diameter makes it potentially well suited for peripheral embolectomy cases. In this report, we demonstrate the use of a $6 \times 30 \mathrm{~mm}$ Solitaire device to revascularize the superior mesenteric artery in a patient with acute mesenteric ischemia. 